\title{
Consumption of dairy products and cardiovascular disease risk: results from the French prospective cohort NutriNet-Santé
}

\author{
Laury Sellem ${ }^{1}$, Bernard Srour ${ }^{2}$, Kim Jackson ${ }^{1}$, Serge Hercberg ${ }^{2}$, Pilar Galan ${ }^{2}$, \\ Emmanuelle Kesse-Guyot ${ }^{2}$, Chantal Julia ${ }^{2}$, Léopold Fezeu ${ }^{2}$, Mélanie Deschasaux ${ }^{2}$, \\ Julie Lovegrove ${ }^{1}$ and Mathilde Touvier ${ }^{2}$ \\ ${ }^{1}$ Hugh Sinclair Unit of Human Nutrition, University of Reading, Reading, United Kingdom and \\ ${ }^{2}$ Epidemiology and Statistics Research Center (CRESS), Inserm U1153, Inra U1125, Cnam, Paris 13 University, \\ Nutritional Epidemiology Research Team (EREN), Bobigny, France
}

\begin{abstract}
Current French National Health and Nutrition Plan (PNNS) recommends 2 servings of dairy products per day for adults. However, dairy contributes to dietary saturated fat intake, of which reduced consumption is often recommended for cardiovascular disease (CVD) prevention. Epidemiological evidence on the association between dairy product consumption and CVD risk remains unclear, with findings from recent prospective cohorts suggesting either null or inverse associations between dairy intake and CVD risk ${ }^{(1,2)}$. This study aimed to investigate the associations between intakes of dairy products (overall and specific types) and CVD risk in a large cohort of French adults.

This prospective study included self-selected participants aged $\geq 18$ years from the NutriNet-Santé cohort (2009-2019). Dietary data were collected every 6 months using 24 h-dietary records, averaged in daily intakes and coded as sex-specific quartiles. Dairy foods were classified according the PNNS dairy groups: milk, cheese, and yogurts (i.e. yogurts, curd cheese and petit-suisses). Total, fermented and low-fat dairy intakes were also investigated. CVD cases $(n=1,952)$ included cerebrovascular (i.e. stroke and transient ischemic attack, $n=878$ cases) and coronary heart diseases (i.e. myocardial infarction, angina, acute coronary syndrome and angioplasty, $\mathrm{n}=1,219$ cases). Multivariable Cox models were performed to characterize associations and were adjusted for age, gender, without-alcohol energy intake, number of $24 \mathrm{~h}$-dietary records, smoking status, educational level, physical activity, BMI, alcohol intake and family history of CVD.

This analysis included $n=104,805$ French adults with a mean age 42.8 (SD 14.6) years and the mean number of dietary records per subject was 5.7 (SD 3.1). There was no association between total or specific dairy intakes and total CVD or coronary heart disease risks. However, consumption of fermented dairy, such as cheese and yogurts, was associated with a $19 \%$ reduction in the risk of cerebrovascular disease $\left(\mathrm{HR}_{\mathrm{Q} 4 \text { vs. } \mathrm{Q} 1}=0.81[0.66-0.98], \mathrm{p}_{\text {trend }}=0.01\right)$.

Despite being important dietary sources of saturated fat, dairy product consumption was not associated with total CVD or coronary heart disease risks in a large cohort of French adults. However, fermented dairy products may be associated with a lower risk of cerebrovascular diseases. Further observational and interventional studies may be needed to further assess the impact of dairy on CVD risk and to identify potential mechanisms underlying the beneficial effects of fermented dairy products on cerebrovascular disease risk.
\end{abstract}

\section{Conflict of Interest}

There is no conflict of interest.

\section{References}

1. Soedamah-Muthu \& De Goede, (2018) Curr Nutr Rep 7(4), 171-182.

2. Zhang et al. (2019) Crit Rev Food Sci Nutr 1-6. 